\title{
Correction to: Tetraspanins in mammalian reproduction: spermatozoa, oocytes and embryos
}

\author{
Jana Jankovičová ${ }^{\cdot}$ Zdeňka Neuerová ${ }^{2,3}$ • Petra Sečová ${ }^{~}$ Michaela Bartóková ${ }^{\text {}}$. Filipa Bubeníčková ${ }^{4}$. \\ Kateřina Komrsková ${ }^{2,5}$ · Pavla Postlerová ${ }^{2,4}$ • Jana Antalíková1 (1)
}

Published online: 20 June 2020

(c) Springer-Verlag GmbH Germany, part of Springer Nature 2020

\section{Correction to: Medical Microbiology and Immunology https://doi.org/10.1007/s00430-020-00676-0}

In the original article, the legends for the Figs. 1 and 2 published incorrectly. Please find the correct legends for the both Figs. 1 and 2 as below.

The original article has been corrected.

The original article can be found online at https://doi.org/10.1007/ s00430-020-00676-0.

Jana Antalíková

jana.antalikova@savba.sk

1 Laboratory of Reproductive Physiology, Center of Biosciences, Institute of Animal Biochemistry and Genetics, Slovak Academy of Sciences, Bratislava, Slovak Republic

2 Laboratory of Reproductive Biology, Institute of Biotechnology of the Czech Academy of Sciences, BIOCEV, Vestec, Czech Republic

3 Faculty of Science, University of Hradec Králové, Hradec Králové, Czech Republic

4 Department of Veterinary Sciences, Faculty of Agrobiology, Food and Natural Resources, Czech University of Life Sciences Prague, Prague, Czech Republic

5 Department of Zoology, Faculty of Science, Charles University, Prague, Czech Republic 


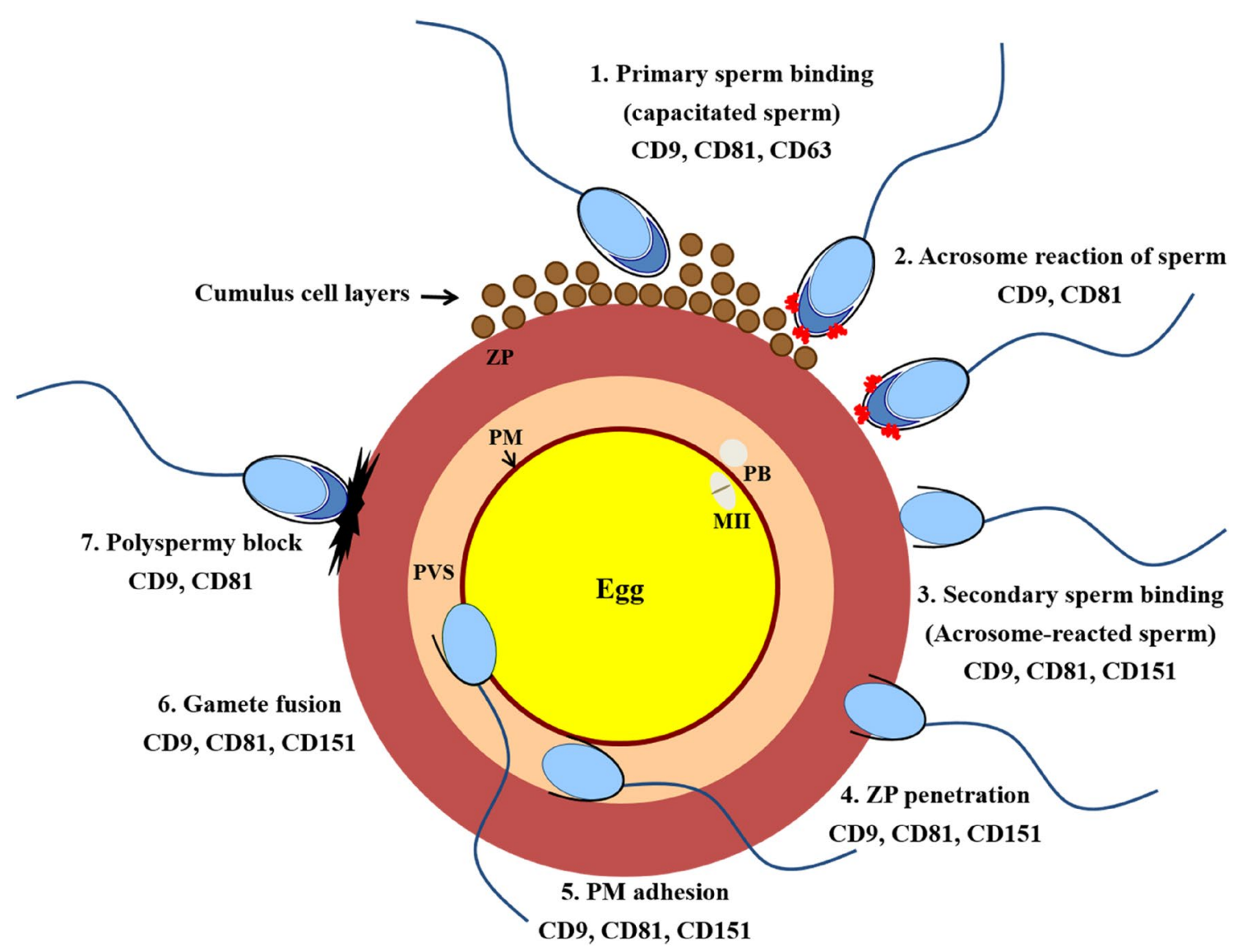

Fig. 1 The engagement of tetraspanins during the sperm and egg interaction. 1. Recognition and primary binding of capacitated sperm to the cumulus cells (CC) and zona pellucida ( $\mathrm{ZP})$ of the egg is assisted by CD9, CD81 and CD63 and followed by 2. the sperm acrosome reaction and CD9 and CD81 relocation (mouse) that facilitates 3. a secondary sperm binding in presence of CD9, CD81 (mouse, human) and CD151 (mouse, human, cattle). This result in 4 . the sperm penetration through ZP and 5. adhesion to the oocyte plasma membrane (PM) both in assistance of CD9, CD81 and CD151. Finally, 6. in presence of CD9, CD81 and CD151 gamete membrane fusion occurs followed by pronuclei fusion and a zygote formation. 7 . shortly after a sperm fuses with the oocyte, the impermeability of $\mathrm{ZP}$ to other sperm is ensured by the polyspermy block. $P B$ polar body, $P V S$ perivitelline space 
Fig. 2 Localization of tetraspanins in mouse, bull and human spermatozoa. The diagram presents the localization of CD9 (green), CD81 (red) and CD151 (blue) in mouse, bull and human spermatozoa before and after the acrosome reaction. Plasma membrane (PM), outer acrosomal membrane (OAM), inner acrosomal membrane (IAM) and equatorial region (ER)

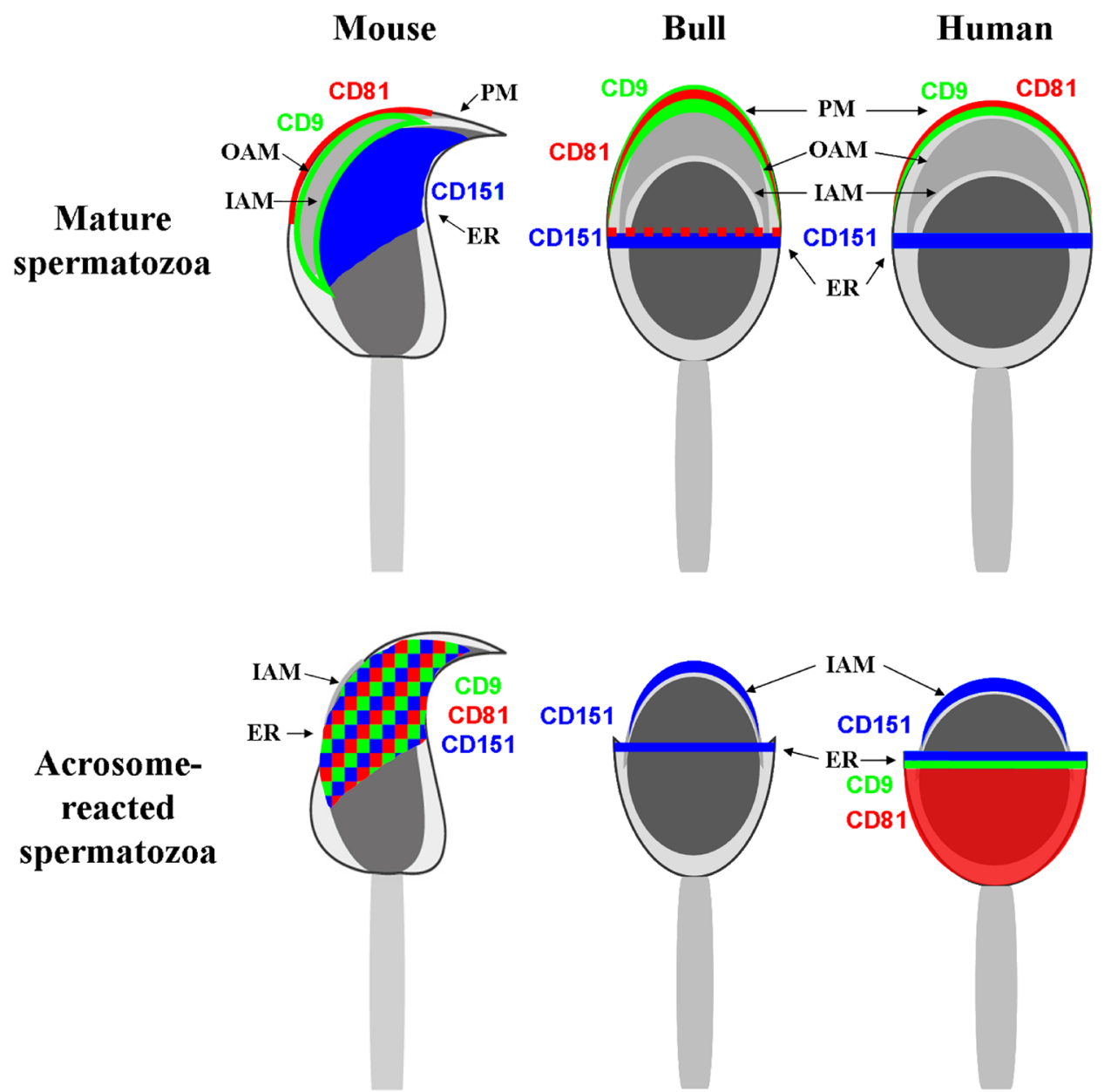

Publisher's Note Springer Nature remains neutral with regard to jurisdictional claims in published maps and institutional affiliations. 